\title{
Pump-Probe Ghost Imaging with SASE FELs
}

\author{
D. Ratner, J. P. Cryan, T. J. Lane, S. Li, and G. Stupakov \\ SLAC National Accelerator Laboratory, Menlo Park, California 94025, USA
}

(Received 25 October 2018; published 11 March 2019)

\begin{abstract}
In this paper, we propose a new approach to measuring ultrafast dynamics with free-electron lasers (FELs). Ultrafast experiments are among the most promising avenues of research at x-ray FELs, with the potential to reveal the chemical dynamics of charge separation, conical intersection crossing, and biologically mediated reactions. Pump-probe scanning is the standard approach to measure dynamics at X-ray FELs, but at the shortest timescales, and particularly for x-ray pump, x-ray probe experiments, the scans require challenging beam setups and can introduce systematic errors. Here, we propose an alternative approach using the randomness of the self-amplified spontaneous emission (SASE) process to drive many simultaneous pump-probe experiments on each shot. Measuring the fluctuations in the incident beam's time profile on a shot-to-shot basis enables the reconstruction of ultrafast dynamics down to the coherence length of the FEL without the need for pump-probe scans. Because of similarity to ghost imaging, in which spatial properties are reconstructed by measuring the incident probe's transverse properties, we call this "pump-probe ghost imaging." In this paper, we describe the method and simulate an example experiment. We also describe an alternative implementation that uses only spectral measurements, avoiding the need for direct time-domain diagnostics and extending the method to the attosecond regime.
\end{abstract}

DOI: 10.1103/PhysRevX.9.011045

One of the most valuable qualities of $\mathrm{x}$-ray free-electron lasers (XFELs) is the ability to probe ultrafast dynamics down to the femtosecond (fs) scale. In the most common "pump-probe" experiments, one photon "pumps" a sample into a nonequilibrium state, and a second photon later "probes" the sample to measure the evolution of that state. Optical pump, $\mathrm{x}$-ray probe measurements are now routinely used to follow chemical reactions at the femtosecond timescales intrinsic to molecular motion, an example of so-called "femtochemistry" [1]. An important subclass of these experiments is the $\mathrm{x}$-ray pump, x-ray probe method, in which both photons are at x-ray energies. For example, a number of recent works exploit the interaction of soft $\mathrm{x}$-ray pulses with core-level electrons to probe excitations in gas phase molecular systems with atomic-site specificity [2-4]. X-ray pump, x-ray probe setups are also relevant to nanocrystallography [5]; while such experiments are ideally "damage-free" [6], careful studies have shown that structural changes in proteins due to radiation-induced rearrangements may survive, highlighting the need to measure $\mathrm{x}$-ray-induced changes at sub-20-fs timescales $[7,8]$.

\footnotetext{
*dratner@slac.stanford.edu
}

Published by the American Physical Society under the terms of the Creative Commons Attribution 4.0 International license. Further distribution of this work must maintain attribution to the author(s) and the published article's title, journal citation, and DOI.

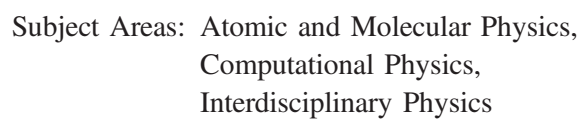

Despite the success of $\mathrm{x}$-ray pump-probe studies, these techniques still suffer from time-intensive machine setups, challenging systematics, and a resolution that rarely exceeds $10 \mathrm{fs}$. To address these issues, here we propose a new method that exploits the chaotic nature of selfamplified spontaneous emission (SASE) XFEL pulses to extract dynamics in the fs regime. Because the SASE FEL process originates from shot noise, each pulse consists of a random train of uncorrelated spikes (see, e.g., Ref. [9]). By measuring the random substructure of individual shots in a sequence of SASE pulses and correlating these to static sample measurements, we demonstrate that it is possible to reconstruct the time evolution of the system at timescales as short as the SASE coherence length, typically much shorter than the duration of the FEL pulse. We emphasize that this method can be implemented with standard single-pulse operation modes and requires no pump-probe scanning; the shot-to-shot variation is provided entirely by the randomness inherent to the SASE process.

Our proposed approach is analogous to classical ghost imaging (GI). Typical GI setups split an illumination beam into two arms, one passing through a sample to a bucket detector and a second "reference" arm measured by a pixelated detector. Correlating fluctuations in the bucket detector to fluctuations at each pixel in the reference arm then reveals a "ghost" image of the sample. (See, e.g., Refs. [10-12] for examples of x-ray GI.) In the standard application, the pixels represent transverse positions in 
the beam. However, by measuring fluctuations in power rather than position, GI can also reveal temporal properties of the sample [13]. Our proposal is similar, except that we measure fluctuations in delay, the relevant quantity for pump-probe experiments. Consequently, we refer to this method as pump-probe ghost imaging (PPGI).

It is instructive to compare PPGI to the wealth of ultrafast methods employed at FELs. For example, the Linac Coherent Light Source (LCLS) has developed several two-pulse pump-probe SASE modes [14-16], and equivalent methods exist for seeded FELs as well $[17,18]$. Recent advances have pushed individual pulse lengths to the subfemtosecond regime with hard $\mathrm{x}$ rays $[19,20]$, and the enhanced-SASE (ESASE) method can reach a similar duration with soft $\mathrm{x}$ rays [21]. We note each of these methods requires a potentially time-intensive setup of a special operating mode, coupled with pump-probe scanning, which can introduce systematic errors. At the freeelectron laser in Hamburg (FLASH), an alternative method makes similar use of the chaotic nature of SASE spikes to extract dynamics at timescales shorter than the pulse length [22]. However, the FLASH technique still requires pumpprobe scanning as well as a split-and-delay line to create a replica pulse, a challenge in the $\mathrm{x}$-ray regime. In contrast to all of the above methods, PPGI measurements are single pulse and passive, requiring only the noninvasive measurement of the incident $\mathrm{x}$-ray beam. As a result, PPGI is well suited as a parasitic addition to standard FEL experiments, for example, monitoring for damage in nanoxtal studies, and as a complementary method for attosecond schemes. PPGI also resembles the Hadamard transform method used at synchrotrons [23]. In the Hadamard transform, a time pattern of the synchrotron is modulated with a predetermined pattern, which is not feasible in the fs regime. By contrast, PPGI exploits the shot-to-shot variations inherent to the SASE process.

We start by considering a two-photon experiment: A pump photon induces a nonequilibrium process, and a probe photon arrives after a delay $\tau$ to measure some property of the sample $\boldsymbol{r}(\tau)$. For example, this modality can reveal the dynamics of core-ionized, gas-phase molecules as follows: The pump step ejects a localized core-electron, resulting in a highly excited molecular cation. This highly excited state decays via the Auger process, which results in the population of multiple dicationic states, each with equilibrium structures that differ from the initially occupied ground state. Following the Auger process, the molecular structure starts to evolve, and a second x-ray photon with energy $\hbar \omega_{0}$ interacts with the new time-dependent species and produces photoelectrons. Measuring the probe photoelectrons' kinetic energy $K_{E}$ reveals the core-level binding energy $\left[E_{B}=\hbar \omega_{0}-K_{E} \equiv \boldsymbol{r}(\tau)\right]$ as a function of the interpulse separation $(\tau)$. This time-resolved x-ray photoelectron spectroscopy has been shown to provide a sensitive probe of the dynamics in excited-state systems [24-26]. Similar x-ray pump-probe schemes are used for a wide variety of science at XFELs (see, e.g., Refs. [27-29]), among them measuring time-dependent change in the electron density, i.e., damage, in nanocrystallography experiments [6].

Traditionally, pump-probe experiments use two distinct "pulses," short bursts of radiation separated in time by $\tau$. Here, we instead use a single pulse, varying in intensity over time, which is responsible for both pump and probe signals, as shown in Fig. 1(a). Effectively, each pulse generates multiple, overlapping, pump-probe measurements at different delays. In the simplest implementation, we make the following additional assumptions:

(1) The probability of a pump photon inducing an excitation is proportional to the instantaneous power.

(2) The probability of a probe photon generating a measurable signal is proportional to the instantaneous power.

These conditions hold broadly for many XFEL experiments. We note that "excitation" does not necessarily refer to electronic states but could include, for example, a damage event to a protein. While these assumptions allow a simple formulation of the problem, PPGI can be applied even if one or both are violated.

We can then write the ensemble distribution of states $\boldsymbol{s}(t)$ at a time $t$ in the "ith" pulse as

$$
\boldsymbol{s}_{i}(t)=\int_{-\infty}^{t} \boldsymbol{p}_{i}\left(t^{\prime}\right) \boldsymbol{r}\left(t-t^{\prime}\right) d t^{\prime}
$$

Here, $t^{\prime}$ is the time at which an individual member in the population is excited, and $\boldsymbol{p}_{i}(t)$ is the power profile of the pulse. In atomic and molecular systems, $\boldsymbol{r}(\tau)$ evolves over short timescales, and we can measure only a time-averaged version of $s_{i}(t)$, which we call $m_{i}$. With the second assumption that the measured signal $m_{i}$ is proportional to the $\mathrm{x}$-ray power, we find

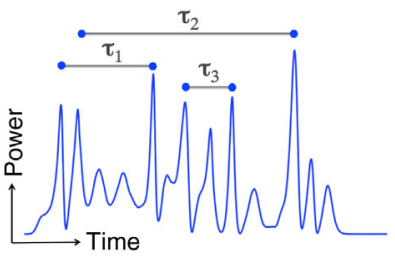

(a)

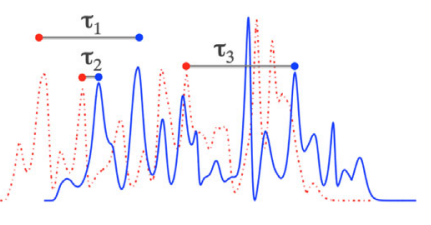

(b)
FIG. 1. (a) The simplest realization with a single SASE pulse: Photons separated by different delays (e.g., $\tau_{1}, \tau_{2}$, and $\tau_{3}$ ) effectively drive independent pump-probe experiments within the same pulse. (b) More complicated setups are also possible, for example, pumping with one wavelength (red dashed line) and probing with another (blue solid line). In both cases, the temporal resolution of the reconstruction is determined by the spike width or measurement resolution and not the pulse duration as in a traditional pump-probe setup. 


$$
m_{i}=\int_{-\infty}^{\infty} \boldsymbol{p}_{i}(t) \boldsymbol{s}_{i}(t) d t=\int_{0}^{\infty} \boldsymbol{a}_{i}(\tau) \boldsymbol{r}(\tau) d \tau
$$

where we use shorthand for the autocorrelation, $\boldsymbol{a}_{i}(\tau) \equiv$ $\int_{-\infty}^{\infty} \boldsymbol{p}_{i}(t) \boldsymbol{p}_{i}(t-\tau) d t$. Our goal is to recover $\boldsymbol{r}(\tau)$ from the measurements $m_{i}$ and $\boldsymbol{a}_{i}(\tau)$.

In a practical implementation with $N_{m}$ pulses and $N_{\tau}$ discretized delays, the autocorrelations form a matrix $\boldsymbol{A} \in \mathbb{R}^{N_{m} \times N_{\tau}}$. Likewise, the individual measurements $m_{i}$ comprise a column vector $\boldsymbol{m} \in \mathbb{R}^{N_{m}}$. Then we may write

$$
m=A r
$$

where $\boldsymbol{r} \in \mathbb{R}^{N_{\tau}}$ is the discretized version of $\boldsymbol{r}(\tau)$. So long as we obtain sufficient pulses of different power profiles, we may infer the underlying system dynamics $\boldsymbol{r}$ by solving this set of linear equations. The experimental schematic is shown in Fig. 2.

The derivation of Eq. (2) implicitly assumes that pump and probe events are independent, which is satisfied if the pump signal does not contribute to $m_{i}$. For example, in the cation experiment described earlier, photoelectrons from the initial pump event are separated by many $\mathrm{eV}$ in energy from those from the probe cation, and the former can be excluded from the bucket detector. However, even when this assumption is violated, PPGI can still be applied; because the pump signal is proportional to the pulse energy rather than the autocorrelation, the pump events will contribute only an uncorrelated noise term to Eq. (3). The presence of additional background increases the number of examples needed for convergence, but it does not affect the identifiability of Eq. (3). The same argument applies to avoiding a signal from multiple excitations.

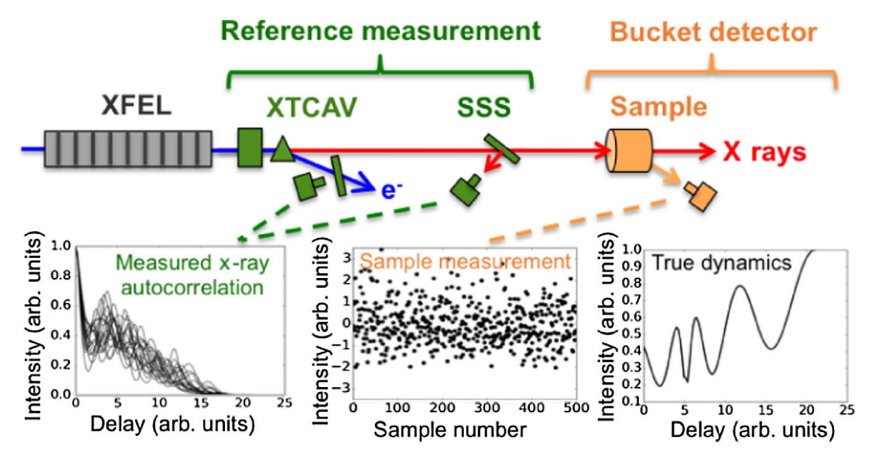

FIG. 2. Schematic of the general PPGI scheme. Time- (e.g., XTCAV) or frequency-domain [e.g., single-shot spectrometer (SSS)] measurements record the autocorrelation on a shot-byshot basis upstream of the sample (lower left). In the terminology of ghost imaging, these reference measurements record the fluctuations incident on the sample. "Bucket" measurements downstream of the sample, e.g., photoelectron counts, contain no explicit time information (lower middle). Correlating shot-to-shot variations in sample measurements with the corresponding measured SASE fluctuations reveals the true sample dynamics (lower right).
In the traditional ghost-imaging formalism, $\boldsymbol{r}$ is found by explicitly calculating the correlation between $\boldsymbol{m}$ and $\boldsymbol{A}$. An alternative compressed sensing formalism finds the most likely solution $\hat{\boldsymbol{r}}$ by optimizing a cost function [30,31], enabling the addition of arbitrary priors to constrain the solution. For example, using L-2 regularized least-squares regression (i.e., Tikhonov or ridge regression), we estimate $r$ by

$$
\hat{\boldsymbol{r}}=\operatorname{argmin}_{\boldsymbol{r}^{\prime}}\left(\left\|\boldsymbol{m}-\boldsymbol{A \boldsymbol { r } ^ { \prime }}\right\|^{2}+\lambda\left\|\boldsymbol{r}^{\prime}\right\|^{2}\right),
$$

with regularization parameter $\lambda$ and vector of fitting parameters $\boldsymbol{r}^{\prime}$. Additional physically inspired constraints, e.g., nonnegativity, $\hat{\boldsymbol{r}} \geq 0$, or causality, $\hat{\boldsymbol{r}}(\tau<0)=0$, can further improve the solution or reduce the required number of measurements, $N_{m}$. Compressed sensing is found to increase the convergence rate for ghost imaging, even allowing reconstruction with fewer samples than pixels (see, e.g., Refs. [32-36]).

For a typical ghost-imaging setup, a beam splitter upstream of the sample sends a portion of the x-ray beam to a reference arm to measure the incident radiation pattern. Because of the challenge of measuring the shot-to-shot power of an XFEL beam, it is easier to infer $\boldsymbol{p}_{i}(t)$ from the spent electron beam, e.g., with an $\mathrm{X}$-band transverse cavity (XTCAV) [37] or with an optical afterburner [38]. In this scenario, the electron beam plays the role of the reference arm (Fig. 2). It may also be possible to directly measure the $\mathrm{x}$-ray beam, for example, by angular streaking [39].

To this point, we assume that it is possible to directly measure the power, $\boldsymbol{p}(t)$, e.g., using an XTCAV. If temporal resolution of the power measurement limits the reconstruction resolution, combining partial time-domain information with spectral measurements enables the refinement of $\boldsymbol{p}(t)$ using phase-retrieval algorithms [40]. Alternatively, we may notice that Eq. (3) requires only the second-order autocorrelation, and there is no need to explicitly measure the power. By the Wiener-Khinchin theorem, the first-order autocorrelation can be calculated directly from the spectral power. For arbitrary fields, there is not a well-determined relation between $\boldsymbol{A}$ and $\boldsymbol{A}^{(f)}$, but, for the special case of a SASE FEL, the two quantities are highly correlated. In Supplemental Material [41], we derive an analytical expression for this correlation in the case of Gaussian wave packets, a common model for an FEL [9]. Figure 3 shows the relation also holds in 1D FEL simulations. Therefore, if we simply treat $\boldsymbol{A}^{(f)}$ as a noisy version of $\boldsymbol{A}$ in Eq. (3), we still expect to recover features of $\boldsymbol{r}$.

Spectral power measurements have several advantages. In general, spectral measurements at XFELs are simpler than those in the time domain; for example, at LCLS, the thin crystal hard x-ray single-shot spectrometer (SSS) [42] provides high-resolution spectra on a shot-by-shot basis simultaneously with sample measurements. Spectrometers 


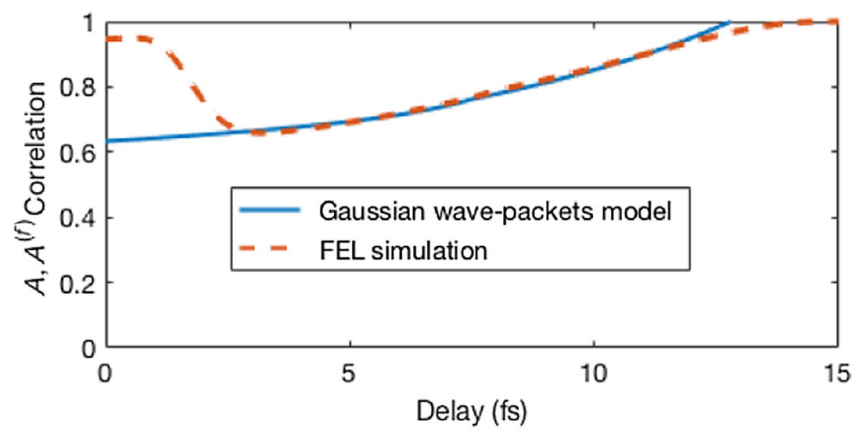

FIG. 3. Fluctuations in $\boldsymbol{A}^{(f)}$ and $\boldsymbol{A}$ are strongly correlated for delays $t_{\text {coh }} \ll \tau<T$, with pulse length $T$ and coherence length $t_{\text {coh }}$. The figure shows the Pearson correlation between $\boldsymbol{A}^{(f)}$ and $\boldsymbol{A}$ for both an analytical model of Gaussian wave packets (solid blue line; see Ref. [41]) and 1D simulations of a presaturation FEL with the parameters in Table I (dashed red line).

can also operate at a higher repetition rate compared to the XTCAV, which runs only at $120 \mathrm{~Hz}$. Finally, the autocorrelation resolution for the spectral approach is limited only by the fluctuations in $\boldsymbol{a}_{i}$, determined by the SASE coherence length $t_{\text {coh }}$. As an example, a hard x-ray FEL such as the LCLS [43] lasing at $1.5 \AA$ has $t_{\mathrm{coh}} \sim 300$ as, giving subfemtosecond resolution without the need for generating ultrashort pulses, pump-probe scanning, or even relative timing of two pulses.

To illustrate the PPGI method, we simulate an experiment with $\boldsymbol{r}$ given by a lineout from the "Eggholder" function, a standard artificial landscape test function. We simulate the X-ray pulses using a 1D FEL code [44] with parameters typical for LCLS soft $\mathrm{x}$-ray operation and running without a taper about 3 gain lengths past saturation, which we find empirically results in the best reconstructions (see Ref. [41], Sec. I). From the power, we calculate the true autocorrelations $\boldsymbol{A}^{*}$ and true signal $\boldsymbol{m}^{*}=\boldsymbol{A}^{*} \times \boldsymbol{r}$. To account for noise and instrument resolution $\Delta_{t}$, we apply Gaussian noise and blurring to $\boldsymbol{A}^{*}$ to produce measurements $\boldsymbol{A}$ and add Gaussian noise to $\boldsymbol{m}^{*}$ to produce measurements $\boldsymbol{m}$. The reconstruction then uses ridge [Eq. (4)] with an additional non-negativity constraint. Table I summarizes the simulated parameters, and Fig. 4 shows the resulting reconstruction. The reconstruction

TABLE I. Parameters for simulations based on LCLS soft x-ray operation. The signal-to-noise ratio is relative to half-max of the signal and is added to $\boldsymbol{M}, \boldsymbol{A}$, and $\boldsymbol{A}^{(f)}$.

$\mathrm{X}$-ray photon energy $(\mathrm{eV})$ 500

X-ray FWHM pulse length $T$ (fs)

X-ray coherence length $t_{\text {coh }}$ (fs)

XTCAV FWHM resolution $\Delta_{t}$ (fs)

SSS FWHM resolution $\Delta_{\nu}(\mathrm{meV})$

Signal-to-noise ratio (FWHM)

Number of shots, $N_{m}$

15

1.6

1 or 4

200

10

$100 \mathrm{k}$

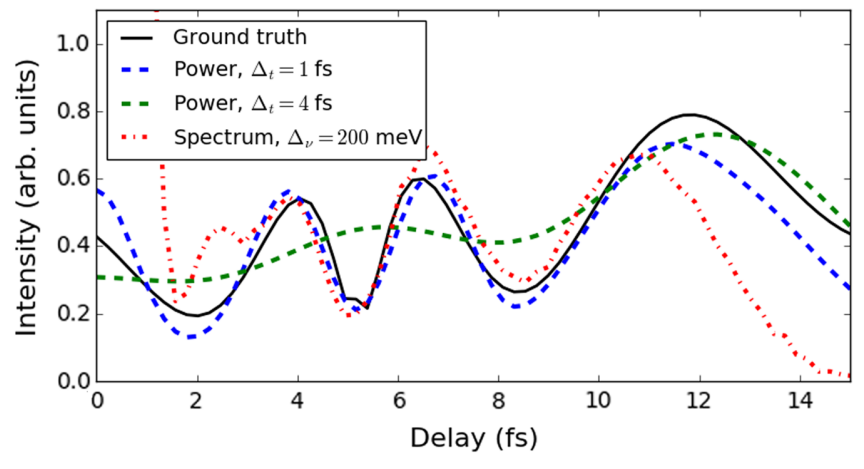

FIG. 4. Illustration of PPGI using simulation parameters from Table I and measurements of either the power (time domain) or spectrum (frequency domain). The ground truth is taken from the Eggholder artificial landscape function.

converges to the ground truth in the range $t_{\text {coh }} \lesssim \tau \lesssim T$, with the resolution limited by the larger of the SASE coherence length $t_{\mathrm{coh}}$ and the instrument resolution. For $\tau \lesssim t_{\text {coh }}$ and $\tau \gtrsim T$, each measurement $\boldsymbol{a}_{i}$ is nearly identical, making $\boldsymbol{A}$ poorly conditioned, and we do not expect to recover $\boldsymbol{r}$ in this range.

We also treat the same problem using the spectral approach. In this case, starting from the simulated spectral power, we add Gaussian noise and blurring from the instrumental resolution $\Delta_{\nu}$ and then calculate the Fourier transform $\boldsymbol{A}^{(f)}$. We shift and scale $\boldsymbol{A}^{(f)}$ so that both the mean and mean fluctuation amplitudes match those of $\boldsymbol{A}$ and use the result in place of $\boldsymbol{A}$ in Eq. (4). We then scale the overall amplitude of $\hat{\boldsymbol{r}}$ to minimize the mean squared error (MSE). Because of the correlation between $\boldsymbol{A}^{(f)}$ and $\boldsymbol{A}$ for FEL radiation, it is still possible to recover a good approximation of $\boldsymbol{r}$ (Fig. 4). Whereas in the time-domain case instrumental resolution limits the reconstruction of sharp features, in the spectral version the spectrometer resolution cuts off reconstruction at long delays but preserves sharp features in the range $2 t_{c} \lesssim \tau \lesssim 3 \hbar / \Delta_{\nu}$.

To this point, we assume each sample measurement is a single scalar. However, XFEL experiments commonly measure a vector of parameters, $\boldsymbol{m}_{i}(y)$, with multiple buckets, $N_{y}$. Here, the $y$ dimension represents a measured experimental parameter, for example, an electron or x-ray spectrum emitted by the sample. In this case, $\boldsymbol{m} \rightarrow \boldsymbol{M} \in \mathbb{R}^{N_{M} \times N_{y}}$ and $\boldsymbol{r} \rightarrow \boldsymbol{R} \in \mathbb{R}^{N_{\tau} \times N_{y}}$. In all other aspects, the problem is identical, and $\boldsymbol{R}$ is still estimated by solving Eq. (4). To demonstrate, Fig. 5 shows a simulation result with $\boldsymbol{R}$ a 2D excerpt from the Eggholder landscape function.

For simplicity, we use a simple least-squares solution. Though sufficient for the example in Fig. 5, more sophisticated approaches may further improve results. For example, we have significant errors on the independent variable, $\boldsymbol{A} \neq \boldsymbol{A}^{*}$, creating what is commonly known as an "errorsin-variables" problem. Least-squares solutions are suboptimal, e.g., due to regression dilution, compared to results 


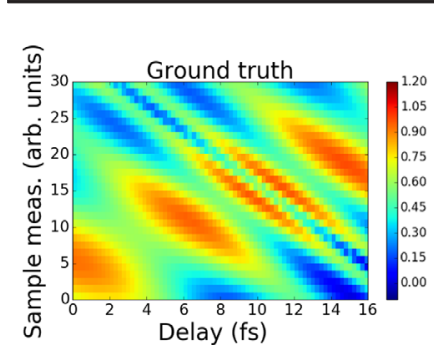

(a)

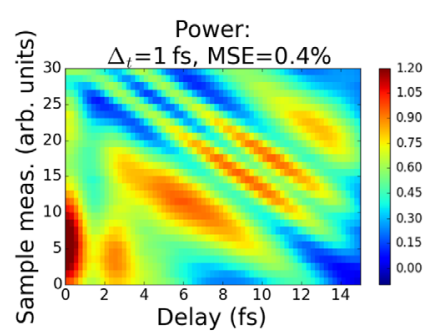

(b)

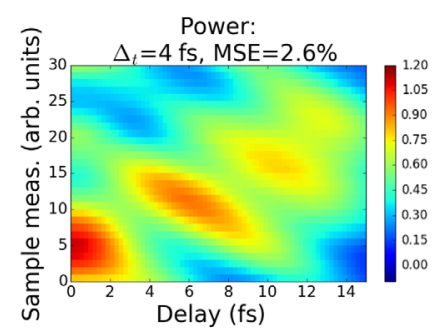

(c)

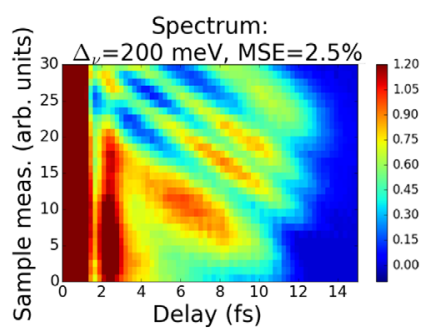

(d)

FIG. 5. (Left) An example taken from the Eggholder artificial landscape function to represent $\boldsymbol{R}$. The $x$ axis corresponds to a pumpprobe delay, and the $y$ axis represents a sample measurement, for example, the intensity of a measured x-ray or electron spectrum emitted by the sample. Reconstructions assume LCLS soft x-ray parameters (Table I) and an instrumental FWHM resolution of $\Delta_{t}=1$ fs (b) and $\Delta_{t}=4 \mathrm{fs}$ (c) for power measurements and $\Delta_{\nu}=200 \mathrm{meV}$ for spectral measurements (d). The MSE is calculated over the range 4-11 fs.

from methods such as total least squares. Second, we retrieve each row of $\boldsymbol{R}$ independently. Modern image analysis tools, for example, the alternating direction method of multipliers [45], enable the simultaneous reconstruction of the entire process, $\boldsymbol{R}$. Simultaneous reconstruction permits the inclusion of additional priors, such as smoothness in both time and energy, that can further improve estimates of $\boldsymbol{R}$.

In this paper, we introduce a new approach to extracting dynamics from XFEL pump-probe experiments. While specialized techniques such as ESASE and nonlinear compression show great promise to reach the attosecond regime, these methods require complex setups [19] and dedicated hardware [20,21] and involve pump-probe scans that introduce systematic error and require precision timing measurements that limit the time resolution. Moreover, the shortest subfemtosecond operating modes [19] are not easily incorporated into pump-probe schemes. By contrast, the ghost-imaging technique presented here needs only a single X-ray pulse, can be performed at any XFEL without modification, can reach subfemtosecond resolution, is equally applicable to hard and soft $\mathrm{x}$ rays, and does not require any special beam tuning. As a passive scheme that does not rely on time scans, PPGI is well suited as a parasitic measurement, e.g., for monitoring nanocrystallography damage [6,8]. With sufficient data, PPGI can recover the short-delay (and thus damage-free) Bragg intensities. Spectral PPGI, though noisier and limited to linear systems, may be the easiest to test experimentally, as it requires only a spectrometer. It also may be possible to improve the temporal resolution by reducing the FEL coherence length, e.g., using ESASE [21] or the microbunching instability $[46,47]$.

While we focus on the simplest case of a single pulse, the method is easily generalized to more complex setups including two-color x-ray schemes (e.g., Refs. [14,15]) or even an optical-pump, X-ray probe [48] with the addition of a timing system [49] [Fig. 1(b)]. Finally, while we assume each sample has been pumped a single time, we can also relax that assumption and allow for multiple interactions between the target and incident x-ray field (complementing, e.g., four-wave mixing protocols [50]). Given that multiphoton excitations are inherently rare events, such schemes will benefit greatly from the increased brightness of the superconducting XFEL facilities currently under development.

We thank Zhirong Huang, Tim Maxwell, and Gordon Wetzstein for their help. This work was supported by U.S. Department of Energy Office of Science under Contract No. DE-AC02-76SF00515. J. P. C.'s contribution is supported by U.S. Department of Energy (DOE), Office of Science, Basic Energy Sciences (BES), Chemical Sciences, Geosciences, and Biosciences Division.

[1] A. Zewail, Femtochemistry: Atomic-Scale Dynamics of the Chemical Bond, J. Phys. Chem. A 104, 5660 (2000).

[2] T. J. A. Wolf et al., Probing Ultrafast $\pi \pi * / n \pi *$ Internal Conversion in Organic Chromophores via k-Edge Resonant Absorption, Nat. Commun. 8, 29 (2017).

[3] A. R. Attar, A. Bhattacherjee, C. D. Pemmaraju, K. Schnorr, K. D. Closser, D. Prendergast, and S. R. Leone, Femtosecond X-Ray Spectroscopy of an Electrocyclic RingOpening Reaction, Science 356, 54 (2017).

[4] Y. Pertot, C. Schmidt, M. Matthews, A. Chauvet, M. Huppert, V. Svoboda, A. von Conta, A. Tehlar, D. Baykusheva, J.-P. Wolf, and H. J. Worner, Time-Resolved $X$-Ray Absorption Spectroscopy with a Water Window High-Harmonic Source, Science 355, 264 (2017).

[5] H. N. Chapman et al., Femtosecond X-Ray Protein Nanocrystallography, Nature (London) 470, 73 (2011).

[6] A. Barty et al., Self-Terminating Diffraction Gates Femtosecond X-Ray Nanocrystallography Measurements, Nat. Photonics 6, 35 (2012).

[7] L. Galli et al., Electronic Damage in s Atoms in a Native Protein Crystal Induced by an Intense X-Ray Free-Electron Laser Pulse, Struct. Dyn. 2, 041703 (2015).

[8] K. Nass et al., Indications of Radiation Damage in Ferredoxin Microcrystals Using High-Intensity X-FEL Beams, J. Synchrotron Radiat. 22, 225 (2015).

[9] K.-J. Kim, Z. Huang, and R. Lindberg, Synchrotron Radiation and Free-Electron Lasers (Cambridge University Press, Cambridge, England, 2017). 
[10] D. Pelliccia, A. Rack, M. Scheel, V. Cantelli, and D. M. Paganin, Experimental X-Ray Ghost Imaging, Phys. Rev. Lett. 117, 113902 (2016).

[11] H. Yu, R. Lu, S. Han, H. Xie, G. Du, T. Xiao, and D. Zhu, Fourier-Transform Ghost Imaging with Hard X Rays, Phys. Rev. Lett. 117, 113901 (2016).

[12] A. M. Kingston, D. Pelliccia, A. Rack, M. P. Olbinado, Y. Cheng, G. R. Myers, and D. M. Paganin, Ghost Tomography, Optica 5, 1516 (2018).

[13] P. Ryczkowski, M. Barbier, A. T. Friberg, J. M. Dudley, and G. Genty, Ghost Imaging in the Time Domain, Nat. Photonics 10, 167 (2016).

[14] A. A. Lutman, R. Coffee, Y. Ding, Z. Huang, J. Krzywinski, T. Maxwell, M. Messerschmidt, and H.-D. Nuhn, Experimental Demonstration of Femtosecond Two-Color X-Ray Free-Electron Lasers, Phys. Rev. Lett. 110, 134801 (2013).

[15] A. Marinelli, D. Ratner, A. A. Lutman, J. Turner, J. Welch, F.-J. Decker, H. Loos, C. Behrens, S. Gilevich, A. A. Miahnahri, S. Vetter, T. J. Maxwell, Y. Ding, R. Coffee, S. Wakatsuki, and Z. Huang, High Intensity Double Pulse X-Ray Free-Electron Laser, Nat. Commun. 6, 6369 (2015).

[16] A. A. Lutman, T. J. Maxwell, J. P. MacArthur, M. W. Guetg, N. Berrah, R. N. Coffee, Y. Ding, Z. Huang, A. Marinelli, S. Moeller, and J.C. U. Zemella, Fresh-Slice Multicolour X-Ray Free-Electron Lasers, Nat. Photonics 10, 745 (2016).

[17] E. Allaria et al., Two-Stage Seeded Soft-X-Ray FreeElectron Laser, Nat. Photonics 7, 913 (2013).

[18] D. Gauthier, P. R. Ribic, G. DeNinno, E. Allaria, P. Cinquegrana, M. B. Danailov, A. Demidovich, E. Ferrari, and L. Giannessi, Generation of Phase-Locked Pulses from a Seeded Free-Electron Laser, Phys. Rev. Lett. 116, 024801 (2016).

[19] S. Huang, Y. Ding, Y. Feng, E. Hemsing, Z. Huang, J. Krzywinski, A. A. Lutman, A. Marinelli, T. J. Maxwell, and D. Zhu, Generating Single-Spike Hard X-Ray Pulses with Nonlinear Bunch Compression in Free-Electron Lasers, Phys. Rev. Lett. 119, 154801 (2017).

[20] A. Marinelli, J. MacArthur, P. Emma, M. Guetg, C. Field, D. Kharakh, A. A. Lutman, Y. Ding, and Z. Huang, Experimental Demonstration of a Single-Spike Hard-X-Ray FreeElectron Laser Starting from Noise, Appl. Phys. Lett. 111, 151101 (2017).

[21] A. A. Zholents and W. M. Fawley, Proposal for Intense Attosecond Radiation from an X-Ray Free-Electron Laser, Phys. Rev. Lett. 92, 224801 (2004).

[22] K. Meyer, C. Ott, P. Raith, A. Kaldun, Y. Jiang, A. Senftleben, M. Kurka, R. Moshammer, J. Ullrich, and T. Pfeifer, Noisy Optical Pulses Enhance the Temporal Resolution of Pump-Probe Spectroscopy, Phys. Rev. Lett. 108, 098302 (2012).

[23] B. A. Yorke, G. S. Beddard, R. L. Owen, and A. R. Pearson, Time-Resolved Crystallography Using the Hadamard Transform, Nat. Methods 11, 1131 (2014).

[24] S. Hellmann, C. Sohrt, M. Beye, T. Rohwer, F. Sorgenfrei, M. Marczynski-Buhlow, M. Kallane, H. Redlin, F. Hennies, M. Bauer, A. Fohlisch, L. Kipp, W. Wurth, and K. Rossnagel, Time-Resolved X-Ray Photoelectron Spectroscopy at FLASH, New J. Phys. 14, 013062 (2012).

[25] A. Shavorskiy et al., Time-Resolved X-Ray Photoelectron Spectroscopy Techniques for Real-Time Studies of
Interfacial Charge Transfer Dynamics, AIP Conf. Proc. 1525, 475 (2013).

[26] C. E. Liekhus-Schmaltz et al., Ultrafast Isomerization Initiated by X-Ray Core Ionization, Nat. Commun. 6, 8199 (2015).

[27] K. R. Ferguson et al., Transient Lattice Contraction in the Solid-to-Plasma Transition, Sci. Adv. 2, e1500837 (2016).

[28] I. Inoue, Y. Inubushi, T. Sato, K. Tono, T. Katayama, T. Kameshima, K. Ogawa, T. Togashi, S. Owada, Y. Amemiya, T. Tanaka, T. Hara, and M. Yabashi, Observation of Femtosecond X-Ray Interactions with Matter Using an X-Ray-X-Ray Pump-Probe Scheme, Proc. Natl. Acad. Sci. U.S.A. 113, 1492 (2016).

[29] C. A. Stan et al., Liquid Explosions Induced by X-Ray Laser Pulses, Nat. Phys. 12, 966 (2016).

[30] E. J. Candes, J. Romberg, and T. Tao, Robust Uncertainty Principles: Exact Signal Reconstruction from Highly Incomplete Frequency Information, IEEE Trans. Inf. Theory 52, 489 (2006).

[31] O. Katz, Y. Bromberg, and Y. Silberberg, Compressive Ghost Imaging, Appl. Phys. Lett. 95, 131110 (2009).

[32] L. Jiying, Z. Jubo, L. Chuan, and H. Shisheng, High-Quality Quantum-Imaging Algorithm and Experiment Based on Compressive Sensing, Opt. Lett. 35, 1206 (2010).

[33] P. Zerom, K. W. C. Chan, J. C. Howell, and R. W. Boyd, Entangled-Photon Compressive Ghost Imaging, Phys. Rev. A 84, 061804 (2011).

[34] V. Katkovnik and J. Astola, Compressive Sensing Computational Ghost Imaging, J. Opt. Soc. Am. A 29, 1556 (2012).

[35] Z. Chen, J. Shi, and G. Zeng, Object Authentication Based on Compressive Ghost Imaging, Appl. Opt. 55, 8644 (2016).

[36] S. Li, F. Cropp, K. Kabra, T. J. Lane, G. Wetzstein, P. Musumeci, and D. Ratner, Electron Ghost Imaging, Phys. Rev. Lett. 121, 114801 (2018).

[37] C. Behrens, F.-J. Decker, Y. Ding, V. A. Dolgashev, J. Frisch, Z. Huang, P. Krejcik, H. Loos, A. Lutman, T. J. Maxwell, J. Turner, J. Wang, M.-H. Wang, J. Welch, and J. Wu, Few-Femtosecond Time-Resolved Measurements of X-Ray Free-Electron Lasers, Nat. Commun. 5, 3762 (2014).

[38] E. L. Saldin, E. A. Schneidmiller, and M. V. Yurkov, Optical Afterburner for an X-Ray Free Electron Laser as a Tool for Pump-Probe Experiments, Phys. Rev. Accel. Beams 13, 030701 (2010).

[39] N. Hartmann et al., Attosecond Time-Energy Structure of X-Ray Free-Electron Laser Pulses, Nat. Photonics 12, 215 (2018).

[40] F. Christie, J. Ransch-Schulenburg, M. Vogt, Y. Ding, Z. Huang, J. Krzywinski, A. A. Lutman, T. J. Maxwell, D. Ratner, and V. A. Jhalani, Temporal X-Ray Reconstruction Using Temporal and Spectral Measurements, in Proceedings of the Ninth International Particle Accelerator Conference (IPAC2018), Vancouver, 2018 (JACoW, Geneva, 2018), TUPMF076.

[41] See Supplemental Material at http://link.aps.org/ supplemental/10.1103/PhysRevX.9.011045 for the Pearson correlation between the first and second order autocorrelations for a SASE FEL and to explore the independence of the SASE autocorrelation matrix.

[42] D. Zhu, M. Cammarata, J. M. Feldkamp, D. M. Fritz, J. B. Hastings, S. Lee, H. T. Lemke, A. Robert, J. L. Turner, and 
Y. Feng, A Single-Shot Transmissive Spectrometer for Hard X-Ray Free Electron Lasers, Appl. Phys. Lett. 101, 034103 (2012).

[43] P. Emma et al., First Lasing and Operation of an AngstromWavelength Free-Electron Laser, Nat. Photonics 4, 641 (2010).

[44] 1D FEL code used courtesy of Zhirong Huang.

[45] S. Boyd, N. Parikh, E. Chu, B. Peleato, and J. Eckstein, Distributed Optimization and Statistical Learning via the Alternating Direction Method of Multipliers, Found. Trends Machine Learning 3, 1 (2011).

[46] E. A. Schneidmiller and M. V. Yurkov, Using the Longitudinal Space Charge Instability for Generation of Vacuum Ultraviolet and X-Ray Radiation, Phys. Rev. Accel. Beams 13, 110701 (2010).

[47] D. Ratner, C. Behrens, Y. Ding, Z. Huang, A. Marinelli, T. Maxwell, and F. Zhou, Time-Resolved Imaging of the
Microbunching Instability and Energy Spread at the Linac Coherent Light Source, Phys. Rev. Accel. Beams 18, 030704 (2015).

[48] J. M. Glownia et al., Time-Resolved Pump-Probe Experiments at the LCLS, Opt. Express 18, 17620 (2010).

[49] M. Harmand, R. Coffee, M. R. Bionta, M. Chollet, D. French, D. Zhu, D. M. Fritz, H. T. Lemke, N. Medvedev, B. Ziaja, S. Toleikis, and M. Cammarata, Achieving FewFemtosecond Time-Sorting at Hard X-Ray Free-Electron Lasers, Nat. Photonics 7, 215 (2013).

[50] F. Bencivenga, R. Cucini, F. Capotondi, A. Battistoni, R. Mincigrucci, E. Giangrisostomi, A. Gessini, M. Manfredda, I. P. Nikolov, E. Pedersoli, E. Principi, C. Svetina, P. Parisse, F. Casolari, M. B. Danailov, M. Kiskinova, and C. Masciovecchio, Four-Wave Mixing Experiments with Extreme Ultraviolet Transient Gratings, Nature (London) 520, 205 (2015). 